\title{
Effect of Cigarette and Shisha smoking on cognitive functions impairment: A cross sectional study
}

\author{
Shahid Bashir ${ }^{1}$, Ghulam Murtaza², \\ Sultan Ayoub $\mathrm{MeO}^{3}$, Abeer Al-Masri ${ }^{4}$
}

\begin{abstract}
Objectives: Cigarette and Shisha smoking is becoming a common practice in young generation worldwide. Since, this is a growing threat to public health, our study aims to investigate the cognitive function responses of cigarette and Shisha inhalation in adolescents.

Methods: This retrospective cross sectional study comprised three groups, cigarette smoker, Shisha smoker, and nonsmoker control group (each $\mathrm{n}=25$ ). All the participants were apparently healthy male volunteers aged 21-24 years. Cognitive functions were assessed by employing "Cambridge Neuropsychological Test Automated Battery". The cognitive functions outcome variables were response time tasks (attention switching task (AST) and the percentage of correct answers pattern recognition memory (PRM) task.

Results: Cigarette and Shisha smokers exhibited a considerable decline in cognitive performance parameters, AST mean correct latency $(p=0.001)$, AST mean correct latency (congruent) $(p=0.001)$, AST mean correct latency (incongruent) $(p=0.001)$ and AST mean correct latency (switching) $(p=0.001)$ compared to matched control group.
\end{abstract}

Conclusions: Cigarette and Shisha smokers exhibited significant impairment in their cognitive functions. The present study findings convince that cigarette and Shisha smokers should quit smoking.

KEYWORDS: Cognitive functions, Shisha, Cigarette, Smoking, CANTAB.

doi: https://doi.org/10.12669/pjms.36.5.2251

How to cite this:

Bashir S, Murtaza G, Meo SA, Al-Masri A. Effect of Cigarette and Shisha smoking on cognitive functions impairment: A cross sectional study. Pak J Med Sci. 2020;36(5):1042-1047. doi: https://doi.org/10.12669/pjms.36.5.2251

This is an Open Access article distributed under the terms of the Creative Commons Attribution License (http://creativecommons.org/licenses/by/3.0), which permits unrestricted use, distribution, and reproduction in any medium, provided the original work is properly cited.

1. Shahid Bashir

Berenson-Allen Centre for non Invasive brain stimulation,

Boston, MA, Harvard Medical School, USA.

2. Ghulam Murtaza

Department of Zoology, University of Gujrat,

Gujrat, Pakistan.

3. Sultan Ayoub Meo

4. Abeer Al-Masri

3,4: Department of Physiology, College of Medicine, King Saud University, Riyadh, Saudi Arabia.

Correspondence:

Prof. Sultan Ayoub Meo, MBBS, PhD.

Professor and Consultant, Department of Physiology,

College of Medicine, King Saud University,

P.O Box 2925 Riyadh-11461,

Kingdom of Saudi Arabia.

E-mail: sultanmeo@hotmail.com smeo@ksu.edu.sa

* Received for Publication:

January 21, 2020

* Revision Received:

* Revision Accepted:

\section{INTRODUCTION}

Cigarette and Shisha smoking is frequently used among young adolescence. ${ }^{1}$ The use of Shisha smoking is about $5 \%-17 \%$ and $6 \%-34 \%$ in American and Middle-Eastern adolescents, respectively. The studies have reported that its prevalence has reached up to $44.3 \%$ in the Middle Eastern countries. ${ }^{2}$ In Shisha smoke, large amount of fruitflavored substances such as "coconut, plum, apple, mango, mint, cola, and strawberry and nicotine, carbon monoxide, fine and ultrafine particulate matter, volatile aldehydes, carcinogenic polycyclic aromatic hydrocarbons, and phenolic compounds are present". Moreover, heavy metals such as lead and arsenic are also present in Shisha smoke ${ }^{3-4}$ which are frequently associated with several diseases. ${ }^{5}$ Cigarette contains 1\%-3\% nicotine while Shisha tobacco has about $2 \%-4 \%$. Moreover, $0.34 \%$ - 
$1.40 \%$ carbon monoxide is present in Shisha smoke. ${ }^{6}$ Presently, electronic cigarettes are also swiftly becoming an alternative form of cigarette smoking worldwide. ${ }^{7}$ Tobacco has been linked with many illnesses, carcinomas ${ }^{8}$ and high mortality. ${ }^{9}$

The trend of using cigarette and Shisha smoking is rapidly increasing in university adolescence worldwide. Therefore, in this study, computerized standard software,"CambridgeNeuropsychological Test Automated Battery (CANTAB)", was employed to investigate the cognitive functions. Its application has an advantage, as it is a computerized test and takes less time doing the task. Moreover, it gives more accurate results especially in tasks requiring counting time and response delay like attention switching task (AST) compared to traditional penand-paper cognitive assessment tasks. "AST and pattern recognition memory (PRM) tests were conducted to investigate the cognitive functions as the "memory and attention" are particularly of the main parameters in assessing cognition in smokers. ${ }^{10}$ Based on modulatory impact of cigarette and Shisha smoking on cognition, we hypothesized that cigarette and Shisha smoking would be linked with decrease in cognitive performance among university adolescents.

\section{METHODS}

This retrospective quasi experimental crosssectional study was carried out in the "College of Medicine, King Saud University, Riyadh, Saudi Arabia" during November 2015-May 2016. Age, gender, ethnicity, and socioeconomic statusmatched design was employed to assess the association between Shisha and cigarette smoking on cognitive functions.

Selection of Subjects: After getting ethical approval, the investigator's team visited the various Shisha smoking cafes and got permission from the owner to interview the Shisha smokers. Selection of participants was based on their volunteer participation. Subjects for this study were enrolled after a detailed interview. Similarly, the cigarette group was selected however; the control group was selected from the university students and research employees.

Exclusion Criteria: All the subjects were asked about their different habits such as Shisha and cigarette smoking, consumption of other tobacco products. Subjects with identified cases of diabetes mellitus, marked obesity, anemia, obstructive lung diseases, malignancy, and chronic alcoholics were excluded. Subjects with known cases of nervousness, difficulty in vision, attention, psychiatric problems, seizures, musculo-skeletal disorders and those on sedatives, hypnotics and who had disturbed sleep history, were excluded from the study. ${ }^{1,7}$ We had three groups ( $\mathrm{n}=25$ in each group) namely cigarette smoker, Shisha smoker, and control (nonsmoker) group. All were male participants with mean age of 23.12 \pm 3.82 (mean \pm standard deviation) years for cigarette smoker group; $24.7 \pm 2.50$ years for Shisha smoker, and $22.9 \pm 3.43$ years for nonsmokers (control) group. All groups were matched for age, ethnicity, gender, and socioeconomic status. In control group, university employees and students were included.

Cognitive function: "CANTAB research suite software, version 6.0.37, Cambridge cognition" was employed to carry out neuropsychological testing. All the tests took 25-30 minutes for completion. The participants sat comfortably on a seat during testing. They were asked to use the index finger of their dominant hand to press the response button.

Attention switching task: This test demonstrates the ability of the participants to switch attention between the position and direction of the arrow. In this test, an arrow was displayed on the screen. The participants were asked to indicate the direction and position of the arrow. This test is used to assess the cognitive functions, which is controlled by prefrontal cortex part of the brain and determines the executive dysfunctioning. Congruent and incongruent trials were performed. In congruent trials, the arrow appeared on the right side of the screen, which pointed to the right. In incongruent trials, the arrow appeared on the right side of the screen but pointed towards the left.

Pattern recognition memory: This test is applied to assess the memory of the participants. In this test, the different visual patterns were displayed on the center of the monitor. The subjects were asked to select one specific pattern among the two patterns displayed on the monitor.

Ethical Approval: The study was approved by the "Review Committee, College of Medicine, King Saud University, Riyadh, Saudi Arabia (CMED-305MB12-2016-17)", and written consent was obtained from the participants.

Statistical Analysis: SPSS software (version 22.0; SPSS Inc., Chicago, Illinois, USA) was used to perform the statistical analysis. Numerical data was expressed as mean, and standard deviation (SD). We used one-way analysis of variance (ANOVA) to compare data from three groups (cigarette smokers, Shisha smokers and nonsmokers). The 
Table-I: Demographic Characteristics.

\begin{tabular}{lcc}
\hline Group & Numbers & Age (Years) (Mean $\pm S D)$ \\
\hline Shisha smoker & 25 & $24.72 \pm 2.50$ \\
Cigarette smoker & 25 & $23.12 \pm 3.82$ \\
Non smoker & 25 & $22.96 \pm 3.43$ \\
\hline
\end{tabular}

SD: Standard deviation.

post-hoc multiple comparison procedures were performed with Tukey's HSD between the groups. The $p$ values less than 0.05 were considered to be statistically significant

\section{RESULTS}

The demographic description of the participants is shown in Table-I. In this study, all the participants were males. The values of "AST mean correct latency, AST mean correct latency (congruent) and AST mean correct latency (incongruent)" were recorded as shown in Fig.1.

The ANOVA revealed a significant main effect for AST congruency cost (mean correct) $(\mathrm{F}=3.406$; $p=0.039)$, AST mean correct latency $(\mathrm{F}=32.859$; $p=0.000)$, AST mean correct latency (congruent) $(\mathrm{F}=33.211 ; p=0.000)$, AST mean correct latency (incongruent) $(\mathrm{F}=29.067 ; p=0.000)$, AST mean correct latency (blocks 3, 5) (non-switching blocks) $(\mathrm{F}=11.641 ; p=0.000)$ and AST mean correct latency (block 7) (switching block) $(\mathrm{F}=41.163 ; p=0.000)$ (Table-II, Figure.1). There was no significant effect among groups for AST switching cost (F $=3.107 ; p=0.051)$, AST percent correct trials $(\mathrm{F}=$ $.554 ; p=0.577)$ and PRM percent correct $(\mathrm{F}=1.773$; $p=0.177$, Table-II).

In Shisha smoker versus control group comparison, there was significant difference for the AST mean correct latency $(p=0.000)$, AST mean

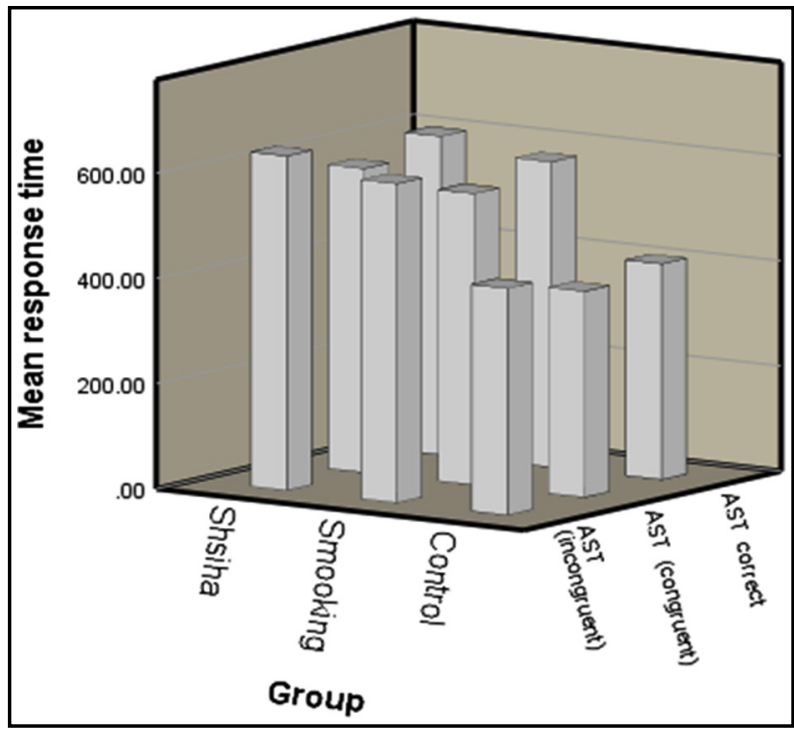

Fig.1: The mean response time (ms) values of AST mean correct latency, AST mean correct latency (congruent) and AST mean correct latency (incongruent) condition for three groups (Shisha smoker, cigarette smoker and control). The error bar showed standard deviation (SD).

correct latency (congruent) $(p=0.000)$, AST mean correct latency (incongruent) $(p=0.000)$, AST mean correct latency (blocks 3, 5) (non-switching blocks) $(p=0.000)$ and AST mean correct latency (block 7) (switching block) $(p=0.000)$ (Table-III). There was not significant effect between two groups for AST congruency cost $(p=0.130)$, AST switching cost $(p=0.980)$, AST percent correct trials $(p=0.641)$ and PRM percent correct ( $p=0.198$, Table-III).

In Cigarette smoker versus control group comparison, there was significant difference for AST congruency cost $(p=0.042)$, AST mean correct latency $(p=0.000)$, AST mean correct latency (congruent) $(p=0.000)$, AST mean correct latency

Table-II: Cognitive function assessment among control, Shisha and cigarette smokers.

\begin{tabular}{lccccc}
\hline Cognitive Functions Parameters & \multicolumn{5}{c}{ Mean \pm SD } \\
\hline & Controls & $\begin{array}{c}\text { Shisha } \\
\text { smokers }\end{array}$ & $\begin{array}{c}\text { Cigarette } \\
\text { smokers }\end{array}$ & F-value & p-value \\
\hline AST congruency cost (mean, correct) & $86 \pm 56$ & $61 \pm 38$ & $55 \pm 36$ & 3.406 & 0.039 \\
AST switching cost (mean, correct) & $304 \pm 122$ & $297 \pm 118$ & $230 \pm 103$ & 3.107 & 0.051 \\
AST mean correct latency & $410 \pm 61$ & $605 \pm 93$ & $580 \pm 114$ & 32.859 & 0.000 \\
AST mean correct latency (congruent) & $391 \pm 53$ & $577 \pm 87$ & $553 \pm 112$ & 33.211 & 0.000 \\
AST mean correct latency (incongruent) & $430 \pm 75$ & $635 \pm 105$ & $606 \pm 121$ & 29.067 & 0.000 \\
AST mean correct latency (blocks 3,5) (non switching blocks) & $369 \pm 55$ & $461 \pm 77$ & $465 \pm 100$ & 11.641 & 0.000 \\
AST mean correct latency (block 7) (switching block) & $450 \pm 77$ & $759 \pm 140$ & $696 \pm 151$ & 41.163 & 0.000 \\
AST percent correct trials & $95 \pm 4$ & 943 & $94 \pm 5$ & .554 & 0.577 \\
PRM percent correct & $81 \pm 7$ & $87 \pm 9$ & $88 \pm 7$ & 1.773 & 0.177 \\
\hline
\end{tabular}

AST mean correct latency ( $\mathrm{p}=0.001)$, AST mean correct latency (congruent) $(\mathrm{p}=0.001)$, AST mean correct latency (incongruent) $(\mathrm{p}=0.001)$ and AST mean correct latency (switching) $(\mathrm{p}=0.001)$ compared to matched control group. 
Table-III: Comparison of various cognitive function test parameters between

Shisha, cigarette smokers compared to their matched control subjects.

\begin{tabular}{|c|c|c|c|}
\hline \multirow[t]{2}{*}{ Cognitive Functions Parameters } & $P(L S D)$ & \multirow[b]{2}{*}{$\begin{array}{l}\text { Cigarette smokers versus } \\
\text { control } 2 \text { versus } 3\end{array}$} & \multirow[b]{2}{*}{$\begin{array}{c}\text { Shisha smokers versus } \\
\text { Cigarette smokers } 1 \text { versus } 3\end{array}$} \\
\hline & $\begin{array}{l}\text { a smokers versus } \\
\text { trol } 1 \text { versus } 2\end{array}$ & & \\
\hline AST congruency cost (mean, correct) & 0.130 & 0.042 & 0.880 \\
\hline AST switching cost (mean, correct) & 0.980 & 0.070 & 0.106 \\
\hline AST mean correct latency & 0.000 & 0.000 & 0.605 \\
\hline AST mean correct latency (congruent) & 0.000 & 0.000 & 0.605 \\
\hline AST mean correct latency (incongruent) & 0.000 & 0.000 & 0.578 \\
\hline $\begin{array}{l}\text { AST mean correct latency (blocks 3, 5) } \\
\text { (non-switching blocks) }\end{array}$ & 0.000 & 0.000 & 0.983 \\
\hline AST mean correct latency (block 7) (switching block) & 0.000 & 0.000 & 0.193 \\
\hline AST percent correct trials & 0.641 & 0.629 & 1.000 \\
\hline PRM percent correct & 0.198 & 0.302 & 0.971 \\
\hline
\end{tabular}

AST: Attention switching task, PRM: Pattern recognition memory, LSD: Least significant difference.

(incongruent) $(p=0.000)$, AST mean correct latency (blocks 3, 5) (non-switching blocks) $(p=0.000)$ and AST mean correct latency (block 7) (switching block) ( $p=0.000$ ) (Table-III). There was no significant effect among two group for AST switching cost $(p=0.070)$, AST percent correct trials $(p=0.629)$ and PRM percent correct ( $p=0.302$, Table-III).

In Shisha smokers versus cigarette smoker groups, values of AST mean correct latency (congruent), AST mean correct latency (incongruent), AST congruency cost, AST mean correct latency, and AST mean correct latency (block 7) (switching block) were higher in Shisha smokers but not significantly different among group. In other tests, values of AST mean correct latency (blocks 3, 5) (non-switching blocks), AST percent correct trials, and PRM percent correct were higher in cigarette smokers but not significantly different Table-III.

\section{DISCUSSION}

Cigarette and Shisha smoking is becoming a common practice in young generation. It severely affects indoor and outdoor air quality and has adverse impact on the human health. In this study, we investigated the impact of Shisha and cigarette smoking on cognitive performance among adolescents and findings from both groups were compared with the results from control group (nonsmokers). It was identified that Shisha and cigarette smoking reduced the cognitive functions compared to nonsmokers. The present study findings reveal that use of Shisha smoking adversely affects attention, speed, and response time. This may be attributed to dysfunctioning of executive functions, which is controlled by frontal lobe of the brain. ${ }^{1}$

Meo et al. ${ }^{1}$ conducted a study on age, education, income, and gender-matched subjects and reported that adult Shisha smokers had significant impairment in their cognitive functions compared to the matched control group. In previous studies, impact of cigarette smoking on cognitive skills has been investigated. For instance, Doiron et al. ${ }^{11}$ demonstrated that cigarette smoking was allied with cognitive impairment. Jacobsen and colleagues et al. ${ }^{12}$ examined the link between cigarette smoking status and various domains of cognitive function in elderly community-dwelling subjects. The cognitive function impairment was demonstrated even in those subjects who had quit cigarette smoking.

Similarly, Fried et al. ${ }^{13}$ conducted a study on age, education, income, and gender-matched subjects and found that cigarette users exhibited significant cognitive impairments on different tested parameters, which included spatial working memory, and executive planning. Glass et al. ${ }^{14}$ demonstrated that compared to nonsmokers, middle-aged male smokers had faster cognitive decline in global cognition and executive function. Timothy et al. ${ }^{15}$ described that cigarette smoking was related to deficiencies in cognitive flexibility, executive functions, working memory, general intellectual abilities, learning and memory processing speed. In line with previous studies, we observed that cigarette and Shisha smoking significantly affected neurocognitive functions.

In cigarette versus control group, there was no significant difference in PRM, which was in accordance with a previous study by Bashir et al. ${ }^{16}$ 


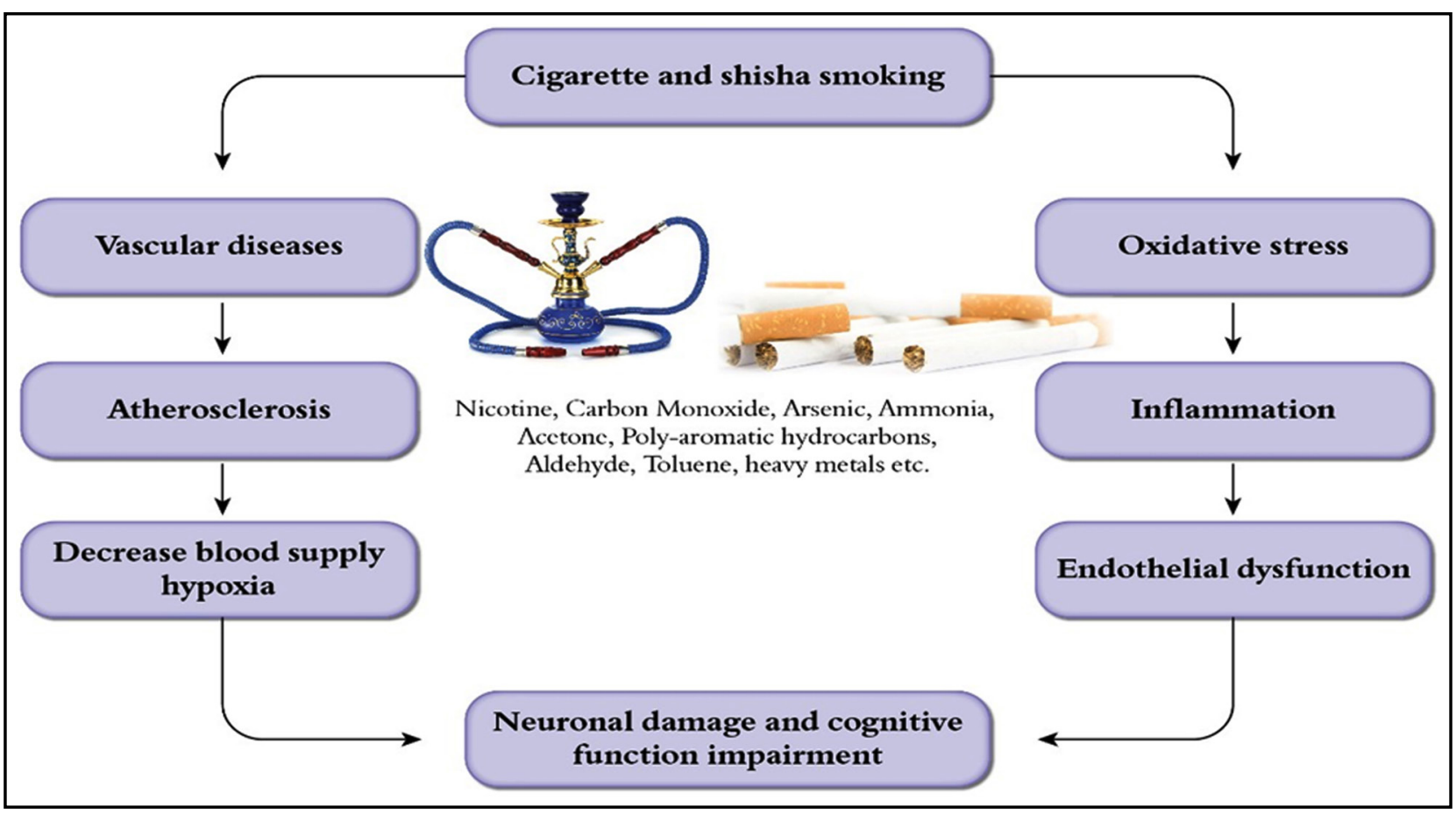

Fig.2: Mechanism how Shisha and cigarette smoking can impair cognitive functions.

This can be attributed to preservation of memory functions in cigarette smokers. In line with our results, cognitive problems due to cigarette smoking in adults have also been demonstrated in various studies.7,11,12 Cigarette smokers showed marked impairment in cognition in sustained attention, spatial working memory, inappropriate behavior, and executive planning domains in comparison with matched nonsmoker group in terms of age, income, gender, and education. ${ }^{11}$ However, we have employed well-validated translational cognitive tasks for evaluation of effects of Shisha and cigarette smoking on cognitive functions, which distinguishes our work from previous studies. The different forms of smoking are rapidly spreading globally, gaining popularity in the Middle East region particularly. With respect to Shisha and cigarette smoking-related investigation on cognitive functions, this work is being reported for the first time in the Middle East region.

The most probable mechanism in line with cigarette, Shisha smoking, and cognitive impairment is the presence of potentially cytotoxic substances which include aldehydes, ketones, carbon monoxide, nitrosamines, dihydroxy benzenes. ${ }^{17}$ These compunds induce oxidation damage to injure the glial or neuronal cells. ${ }^{16,18}$ These cytotoxic compounds may affect the neuronal and cellular membrane function of cerebral hemisphere. It has also been demonstrated that smoking evokes cell injury and causes brain function loss with significant effects in the hippocampus..$^{18}$ In line with the above-mentioned studies, we believe that these are the basic factors behind impairment of cognitive functions due to cigarette and Shisha smoking (Fig.2).

Study strengths and limitations: This work has some strengths and limitations. The major strengths of this study are that, to our best familiarity, no study exists yet, which describes the link between the Shisha and cigarette smoking with cognitive function impairment. The literature is available exploring the neurocognitive consequences of cigarette smoking in middle aged people, there is a specific shortage of studies in the young adults. This is the first study added in the literature on Shisha, cigarette smoking and cognitive functions. The limitation of the present study is the involvement of limited small sample size of Shisha, and cigarette smokers because most of the adolescents were using both Shisha and cigarette, and due to cross-sectional design we could not establish the causation.

\section{CONCLUSIONS}

The young adult cigarette and Shisha smokers exhibited significant impairment in their cognitive functions compared to their matched control 
group. The present study findings would convince that both cigarette and Shisha smokers should quit smoking. Electronic and print media should also play their role in conveying the message that cigarette and Shisha smoking should not be a part of cultural identity, and avoid social acceptability and perception that Shisha is less harmful or less addictive than cigarettes.

Acknowledgements: We thank "Researchers supporting project number (RSP-2019/47), King Saud University, Riyadh, Saudi Arabia".

Declaration of Interests: The authors declare no conflicts of interest.

\section{REFERENCES}

1. Meo SA, Bashir S, Almubarak Z, Alsubaie Y, Almutawa H. Shisha smoking: impact on cognitive functions impairments in healthy adults. Eur Rev Med Pharmacol Sci. 2017;21:52175222. doi: 10.26355/eurrev_201711_13844

2. MacKillop J, Amlung MT, Wier LM, David SP, Ray LA, Bickel WK The neuroeconomics of nicotine dependence: A preliminary functional magnetic resonance imaging study of delay discounting of monetary and cigarette rewards in smokers. Psych Res. 2012;202(1):20-29. doi: 10.1016/j.pscychresns.2011.10.003

3. Mansvelder HD, Van Aerde KI, Couey JJ, Brussaard AB. Nicotinic modulation of neuronal networks: From receptors to cognition. Psychopharmacology (Berl), 2006;184(3-4):292305. doi: $10.1007 /$ s00213-005-0070-z

4. Tuesta LM, Fowler CD, Kenny PJ. Recent advances in understanding nicotinic receptor signaling mechanisms that regulate drug self-administration behavior. Biochem Pharmacol. 2011;82:984-995. doi: 10.1016/j. bcp.2011.06.026

5. Khemiss M, Ben Fekih D, Ben Khelifa M, Ben Saad H. Comparison of Periodontal Status between Male Exclusive Narghile Smokers and Male Exclusive Cigarette Smokers. Am J Mens Health. 2019;13:1557988319839872. doi: $10.1177 / 1557988319839872$.

6. Chamberlain SR, Sahakian BJ. The neuropsychiatry of impulsivity. Curr Opin Psych. 2007;20:255-261. doi: 10.1097/ YCO.0b013e3280ba4989

7. Meo SA, Ansary MA, Barayan FR, Almusallam AS, Almehaid AM, Alarifi NS, et al. Electronic Cigarettes: Impact on Lung Function and Fractional Exhaled Nitric Oxide Among Healthy Adults. Am J Mens Health. 2019;13:1557988318806073. doi: $10.1177 / 1557988318806073$

8. Kanwal M, Haider G, Zareef U, Saleem S. Addiction of tobacco chewing and smoking in the patients of head and neck squamous cell carcinoma: A descriptive epidemiological study in Pakistan. Pak J Med Sci. 2019;35(6):1712-1717. doi: 10.12669/ pjms.35.6.1309
9. Hameed S, Zuberi FF, Hussain S, Ali SK. Risk factors for mortality among inpatients with smear positive pulmonary tuberculosis. Pak J Med Sci. 2019;35(5):1361-1365. doi: 10.12669 / pjms.35.5.919

10. Chamberlain SR, Odlaug BL, SchreiberLR, Grant JE. Association between tobacco smoking and cognitive functioning in young adults. Am J Addict. 2012;21(Suppl1):S14-S19. doi: 10.1111/j.1521-0391.2012.00290.x

11. Doiron $M$, Dupre $N$, Langlois $M$, Provencher $P$, Simard M. Smoking history is associated to cognitive impairment in Parkinson's disease. Aging Mental Health, 2017;21:322-326.

12. Jacobsen LK, Krystal JH, Mencl WE, Westerveld M, Frost SJ, Pugh KR. Effects of smoking and smoking abstinence on cognition in adolescent tobacco smokers. Biolog Psych. 2005;57:56-66. doi: 10.1016/j.biopsych.2004.10.022

13. Fried PA, Watkinson B, Gray R. Neurocognitive consequences of cigarette smoking in young adults- A comparison with pre-drug performance. Neurotoxicol Teratol. 2006;28:517-525. doi: 10.1016/j. ntt.2006.06.003

14. Glass JM, Adams KM, Nigg JT, Wong MM, Puttler LI, Buu A. Smoking is associated with neurocognitive deficits in alcoholism. Drug Alcohol Depend 2006;82:119-126. doi: 10.1111/j.1521-0391.2012.00290.x

15. Timothy C, Durazzo DJ, Meyerhoff S, Nixon J. Chronic cigarette smoking: implications for neuro-cognition and brain neurobiology. Int J Environ Res Public Health. 2010;7:3760-3791.

16. Bashir S, Alghamd F, Alhussien A, Alohali M, Alatawi A, Almusned T. Effect of smoking on cognitive functioning in young Saudi adults. Med Sci Monit Basic Res. 2017;23:31-35. doi: 10.12659/msmbr.902385

17. Zhang XY, Tan YL, Chen DC, Tan SP, Yang FD, ZuntaSoares GB, et al. Effects of cigarette smoking and alcohol use on neuro-cognition and BDNF levels in a Chinese population. Psychopharmacology (Berl). 2016;233:435-445.

18. Kenney JW, Gould TJ. Modulation of hippocampusdependent learning and synaptic plasticity by nicotine. Mol Neurobiol. 2008;38:101-121. doi: 10.1007/s12035008-8037-9

\section{Authors' Contributions:}

SB: Study design, supervised the project, data analysis and manuscript writing.

GM, AAM: Literature review, subject selections. SAM: Technical support and manuscript writing. All authors have approved the final version of the manuscript. 